\title{
Numerical Simulation of Two Dimensional Coupled Burgers Equations by Rubin-Graves Type Linearization
}

\author{
Nuri Murat Yağmurlu*, Abdulnasır Gagir
}

\begin{abstract}
In the present article, the numerical solution of the two-dimensional coupled Burgers equation has been sought by finite difference method based on Rubin-Graves type linearization. Three models with appropriate initial and boundary conditions are applied to the problem. In order to show the accuracy of the method, the error norms $L_{2}, L_{\infty}$ are computed. The error norms $L_{2}, L_{\infty}$ of the obtained numerical solutions are compared with the error norms of some of the numerical solutions in the literature.
\end{abstract}

Keywords: Two-dimensional Burgers equation; Rubin-Graves type linearization; Finite difference method.

AMS Subject Classification (2020): Primary: 35Q51 ; Secondary: 74J35; $33 F 10$.

*Corresponding Author

\section{Introduction}

In nature, some of the pysical phenomena such as gas dynamics, traffic flow, Brusselator chemical reactiondiffusion and shock waves are modelled by nonlinear partial differential equation systems among others such as the two-dimensional coupled Burgers equation (2D-CBE). There are many theoretical and numerical studies about the 2D-CBE equation in the literature. Fletcher [1] has found its analytical solution by applying the two-dimensional Hopf-Cole transform to the two-dimensional coupled Burgers equation. 2D-CBE has been solved numerically by several scholars by means of various methods and techniques. Among others, Fletcher [2] have conducted a work for comparing finite difference and finite element methods. Goyon [3] applied multi level alternating direction implicit methods. Ali et al. [4] have used the collation method via the radial base functions. Jain and Holla [5] have implemented two algorithms using the cubic spline function technique. Bahadır [6] has dealt with the problem by a fully implicit finite difference method. Khater et al. [7] have found out the numerical solution of some Burgers type nonlinear partial differential equations by Chebyshev spectral collocation method.Mittal and Jiwari [8] have applied the differential quadrature method using the Chebyshev-Gauss-Lobatto nodal points. Liao [9] obtained the numerical solution of the two-dimensional coupled Burgers equation by solving the twodimensional linear heat equation obtained by applying the two-dimensional Hopf-Cole transformation to the 
two-dimensional coupled Burgers equation using the fourth-dimensional finite difference method. Zhu et al. [10] applied the discrete Adomian decomposition method. Srivastava et al. have applied [11] Crank-Nicolson finite difference method, Tamsir and Srivastava [12] have used semi-implicit finite difference method, Srivastava and Tamsir [13] have utilized Crank-Nicolson semi-implicit finite difference method, Thakar and Wani [14] have used linear finite difference method, Srivastava et al. [15] have applied implicit logarithmic finite difference method, Srivastava et al. [16] have used implicit exponential finite difference method, Srivastava and Singh [17] have used explicit-implicit finite difference method, Zhang et al. [18] have used full finite difference and non-standard finite difference methods, Mittal and Tripathi [19] have applied modified bi-cubic B-spline collocation method, Tamsir et al. [20] have used exponential modified cubic-B-spline differential quadrature method, Zhanlav et al. [21] have applied high order explicit finite difference method and Ngondiep [22] has utilized three-level explicit time-split MacCormack algorithm. Saqib et al. [23] have dealt with numerical solutions of 2-dimensional time dependent coupled non-linear systems. Wubs and Goede [24], in their article, considered the fully explicit method resulting from the truncation in the solution process and chosen one of the test problems as the 2-dimensional coupled Burgers' equation. Chai and Ouyang [25] have used proper stabilized Galerkin methods.

The rest of this article is organized as follows: In the first section, the method based on Rubin-Graves type linearization together with finite difference method and used for the numerical solution of two dimensional coupled Burgers equation is presented. Then to see the performance accuracy of the method, the numerical solution of three test model problems has been made and presented in tables by calculating the pointwise values and the error norms $L_{2}$ and $L_{\infty}$ of the model problems of which the analytical solution are known. In addition, comparisons have been made with the error norms of the numerical solutions obtained by various methods available in the literature. In the last section, a brief conclusion is given.

\section{Application of the Method}

In this article, we consider the the two-dimensional coupled Burgers equation of the general form given as

$$
\begin{array}{ll}
u_{t}+u u_{x}+v u_{y}=\varepsilon\left(u_{x x}+u_{y y}\right), & (x, y) \epsilon \Omega, t>0 \\
v_{t}+u v_{x}+v v_{y}=\varepsilon\left(v_{x x}+v_{y y}\right), \quad(x, y) \epsilon \Omega, t>0
\end{array}
$$

together with the initial

$$
\begin{array}{ll}
u(x, y, 0)=\psi_{1}(x, y) ; & (x, y) \epsilon \Omega \\
v(x, y, 0)=\psi_{2}(x, y) ; \quad & (x, y) \epsilon \Omega
\end{array}
$$

and the boundary conditions

$$
\begin{array}{ll}
u(x, y, t)=\xi(x, y, t) ; & (x, y) \epsilon \partial \Omega \\
v(x, y, t)=\zeta(x, y, t) ; & (x, y) \epsilon \partial \Omega
\end{array}
$$

where $u(x, y, t)$ and $v(x, y, t)$ denote velocity components. Over the solution domain $\Omega=\{(x, y): a \leqslant x \leqslant b, c \leqslant y \leqslant d\}$ together with its boundary $\partial \Omega . \psi_{1}, \psi_{2}, \xi$ and $\zeta$ are known smooth functions. Re denotes the Reynold number. As it is widely known, at the large values of the Reynold number, a shock wave having a cusp results in and numerical stability near this shock wave is nearly always difficult to obtain.

For the solution process, the domain of the problem in $x$-direction $[a, b]$ is divided into $N_{x}$ parts having equal length $h_{x}$, and in $y$-direction $[c, d]$ is divided into $N_{y}$ parts having equal length $h_{y}, x_{i}=a+i h_{x}, i=0(1) N_{x}$; $y_{j}=c+j h_{y}, j=0(1) N_{y}$; a smooth grid is created in the solution domain of the problem with the help of nodal points $\left(x_{i}, y_{j}\right)$. The step length $\Delta t$ is taken in the direction of the time variable for $t_{n}=n \Delta t, n=0(1) N$,. Then, all the numerical calculations to be made in each time step $t_{n}$ are obtained at the nodes of this smooth grid. The numerical solution of $u(x, y, t)$ and $v(x, y, t)$ at any node $\left(x_{i}, y_{j}, t_{n}\right)$ is shown by $U_{i, j}^{n}$ and $V_{i, j}^{n}$, respectively.

When the finite difference method based on Rubin-Graves type linearization technique is applied, a linear algebraic equation system results in since the related finite difference approaches are written in place of the derivatives in the equation. In the proposed method, the nonlinear partial differential equation is written in the appropriate form and after applying the finite difference method, an iterative relationship between the $(n+1)^{t h}$ and 
$(n)^{t h}$ time level steps of the dependent variables is obtained. This newly obtained iterative relationship resulted in a linear algebraic equation system, which can be easily solved by a symbolic programming language such as MatLab.

Now, for 2D-CBE

$$
\begin{aligned}
u_{t}+u u_{x}+v u_{y} & =\varepsilon\left(u_{x x}+u_{y y}\right) \\
v_{t}+u v_{x}+v v_{y} & =\varepsilon\left(v_{x x}+v_{y y}\right)
\end{aligned}
$$

in place of non-linear terms $u u_{x}, v u_{y}, u v_{x}$ and $v v_{y}$ Rubin-Graves type [26] linearization technique are used.

In place of $u_{t}$ an approximation as $u_{t} \cong\left(U_{i, j}^{n+1}-U_{i, j}^{n}\right) / k$ and in place of $v_{t}$ an approximation as $v_{t} \cong\left(V_{i, j}^{n+1}-\right.$ $\left.V_{i, j}^{n}\right) / k$ and in places of the terms $u u_{x}, v u_{y}, u v_{x}$ and $v v_{y}$ the following Rubin-Graves type approximations

$$
\begin{aligned}
& u u_{x} \cong U_{i, j}^{n+1}\left[\frac{U_{i+1, j}^{n}-U_{i-1, j}^{n}}{2 h_{x}}\right]+U_{i, j}^{n}\left[\frac{U_{i+1, j}^{n+1}-U_{i-1, j}^{n+1}}{2 h_{x}}\right]-U_{i, j}^{n}\left[\frac{U_{i+1, j}^{n}-U_{i-1, j}^{n}}{2 h_{x}}\right] \\
& v u_{y} \cong V_{i, j}^{n+1}\left[\frac{U_{i+1, j}^{n}-U_{i-1, j}^{n}}{2 h_{y}}\right]+V_{i, j}^{n}\left[\frac{U_{i+1, j}^{n+1}-U_{i-1, j}^{n+1}}{2 h_{y}}\right]-V_{i, j}^{n}\left[\frac{U_{i+1, j}^{n}-U_{i-1, j}^{n}}{2 h_{y}}\right] \\
& u v_{x} \cong U_{i, j}^{n+1}\left[\frac{V_{i+1, j}^{n}-V_{i-1, j}^{n}}{2 h_{x}}\right]+U_{i, j}^{n}\left[\frac{V_{i+1, j}^{n+1}-V_{i-1, j}^{n+1}}{2 h_{x}}\right]-U_{i, j}^{n}\left[\frac{V_{i+1, j}^{n}-V_{i-1, j}^{n}}{2 h_{x}}\right] \\
& v v_{y} \cong V_{i, j}^{n+1}\left[\frac{V_{i+1, j}^{n}-V_{i-1, j}^{n}}{2 h_{y}}\right]+V_{i, j}^{n}\left[\frac{V_{i+1, j}^{n+1}-V_{i-1, j}^{n+1}}{2 h_{y}}\right]-V_{i, j}^{n}\left[\frac{V_{i+1, j}^{n}-V_{i-1, j}^{n}}{2 h_{y}}\right]
\end{aligned}
$$

are written. Then in place of the derivatives $u_{x x}, u_{y y}, v_{x x}$ and $v_{y y}$ their central finite difference approximations

$$
\begin{aligned}
& u_{x x} \cong \frac{U_{i-1, j}^{n+1}-2 U_{i, j}^{n+1}+U_{i+1, j}^{n+1}}{h_{x}^{2}} \\
& u_{y y} \cong \frac{U_{i, j-1}^{n+1}-2 U_{i, j}^{n+1}+U_{i, j+1}^{n+1}}{h_{y}^{2}} \\
& v_{x x} \cong \frac{V_{i-1, j}^{n+1}-2 V_{i, j}^{n+1}+V_{i+1, j}^{n+1}}{h_{x}^{2}} \\
& v_{y y} \cong \frac{V_{i, j-1}^{n+1}-2 V_{i, j}^{n+1}+V_{i, j+1}^{n+1}}{h_{y}^{2}}
\end{aligned}
$$

are written. Finally, the terms on the $(n+1)^{t h}$ time level are taken on the left hand side and $(n)^{t h}$ time level terms are taken on the right hand side. After some simpliciation process, the following

$$
\begin{aligned}
& U_{i-1, j}^{n+1}\left(-\frac{k}{2 h_{x}} U_{i, j}^{n}-\frac{\varepsilon k}{h_{x}^{2}}\right)+U_{i, j}^{n+1}\left(1+k\left(\frac{U_{i+1, j}^{n}-U_{i-1, j}^{n}}{2 h_{x}}\right)+4 \frac{\varepsilon k}{h_{x}^{2}}\right) \\
& +U_{i+1, j}^{n+1}\left(\frac{k}{2 h_{x}} U_{i, j}^{n}-\frac{\varepsilon k}{h_{x}^{2}}\right)+U_{i, j-1}^{n+1}\left(-\frac{k}{2 h_{y}} V_{i, j}^{n}-\frac{\varepsilon k}{h_{y}^{2}}\right) \\
& +U_{i, j+1}^{n+1}\left(\frac{k}{2 h_{y}} V_{i, j}^{n}-\frac{\varepsilon k}{h_{y}^{2}}\right)+V_{i, j}^{n+1}\left(\frac{k\left(U_{i, j+1}^{n}-U_{i, j-1}^{n}\right)}{2 h_{y}}\right) \\
& =U_{i, j}^{n}\left[1+k\left(\frac{U_{i+1, j}^{n}-U_{i-1, j}^{n}}{2 h_{x}}\right)\right]+V_{i, j}^{n}\left[k\left(\frac{U_{i, j+1}^{n}-U_{i, j-1}^{n}}{2 h_{y}}\right)\right]
\end{aligned}
$$


and

$$
\begin{aligned}
& V_{i-1, j}^{n+1}\left(-\frac{k}{2 h_{x}} U_{i, j}^{n}-\frac{\varepsilon k}{h_{x}^{2}}\right)+V_{i, j}^{n+1}\left(1+k\left(\frac{V_{i, j+1}^{n}-V_{i, j-1}^{n}}{2 h_{y}}\right)+4 \frac{\varepsilon k}{h_{x}^{2}}\right) \\
& +V_{i+1, j}^{n+1}\left(\frac{k}{2 h_{x}} U_{i, j}^{n}-\frac{\varepsilon k}{h_{x}^{2}}\right)-V_{i, j-1}^{n+1}\left(\frac{k}{2 h_{y}} V_{i, j}^{n}+\frac{\varepsilon k}{h_{y}^{2}}\right) \\
& +V_{i, j+1}^{n+1}\left(\frac{k}{2 h_{y}} V_{i, j}^{n}-\frac{\varepsilon k}{h_{y}^{2}}\right)+U_{i, j}^{n+1}\left(\frac{k\left(V_{i+1, j}^{n}-U_{i-1, j}^{n}\right)}{2 h_{x}}\right) \\
& =V_{i, j}^{n}\left[1+k\left(\frac{V_{i+1, j}^{n}-V_{i-1, j}^{n}}{2 h_{x}}\right)\right]+U_{i, j}^{n}\left[k\left(\frac{V_{i, j+1}^{n}-V_{i, j-1}^{n}}{2 h_{y}}\right)\right]
\end{aligned}
$$

linearized schemes are obtained, where $i, j=1(1) M-1$. In these schemes $h_{x}=h_{y}, \varepsilon k / h_{x}^{2}=\varepsilon k / h_{y}^{2}=a$, $k / 2 h_{x}=k / 2 h_{y}=b$ and $\varepsilon=1 / \operatorname{Re}$ are taken as some simplifications are carried out. Finally, the following

$$
\begin{aligned}
& -U_{i-1, j}^{n+1}\left[b U_{i, j}^{n}+a\right]+U_{i, j}^{n+1}\left[1+4 a+b\left(U_{i+1, j}^{n}-U_{i-1, j}^{n}\right)\right]+U_{i+1, j}^{n+1}\left[b U_{i, j}^{n}-a\right] \\
& \left.-U_{i, j-1}^{n+1}\left[b V_{i, j}^{n}+a\right)\right]+U_{i, j+1}^{n+1}\left[b V_{i, j}^{n}-a\right]+V_{i, j}^{n+1}\left[b\left(U_{i, j+1}^{n}-U_{i, j-1}^{n}\right)\right] \\
& =U_{i, j}^{n}\left[1+b\left(U_{i+1, j}^{n}-U_{i-1, j}^{n}\right)\right]+V_{i, j}^{n}\left[b\left(U_{i, j+1}^{n}-U_{i, j-1}^{n}\right)\right]
\end{aligned}
$$

and

$$
\begin{aligned}
& -V_{i-1, j}^{n+1}\left[b U_{i, j}^{n}+a\right]+V_{i, j}^{n+1}\left[1+4 a+b\left(V_{i, j+1}^{n}-V_{i, j-1}^{n}\right)\right]+V_{i+1, j}^{n+1}\left[b U_{i, j}^{n}-a\right] \\
& \left.-V_{i, j-1}^{n+1}\left[b V_{i, j}^{n}+a\right)\right]+V_{i, j+1}^{n+1}\left[b V_{i, j}^{n}-a\right]+U_{i, j}^{n+1}\left[b\left(V_{i+1, j}^{n}-U_{i-1, j}^{n}\right)\right] \\
& =V_{i, j}^{n}\left[1+b\left(V_{i, j+1}^{n}-V_{i, j-1}^{n}\right)\right]+U_{i, j}^{n}\left[b\left(V_{i+1, j}^{n}-V_{i-1, j}^{n}\right)\right]
\end{aligned}
$$

schemes are obtained. Using the known $U^{n}$ and $V^{n}$ values in the finite difference diagrams obtained as a result of this linearization, the unknown values of $U^{n+1}$ and $V^{n+1}$ at the desired time $t$ are obtained for all three model problems.

\section{Numerical Results}

In this section, the numerical solution of the two-dimensional coupled Burgers equation given by the equations (2.1)-(2.2), for three problems with appropriate initial and boundary conditions using the finite difference method based on Rubin-Graves type linearization has been obtained. In order to show the accuracy of the obtained numerical solutions, the following error norms $L_{2}$ and $L_{\infty}$ are calculated

$$
L_{2}=\sqrt{\sum_{i=1}^{N_{x}-1} \sum_{j=1}^{N_{y}-1}\left|U_{i j}-\left(u_{\text {exact }}\right)_{i j}\right|^{2}}
$$

and

$$
L_{\infty}=\max _{i, j}\left|U_{i, j}-\left(u_{\text {exact }}\right)_{i, j}\right|
$$

where $u_{i j}^{n}$ are analytical solutions and $U_{i j}^{n}$ are approximate solutions at the nodal points $\left(x_{i}, y_{j}, t_{n}\right)$ [27].

Problem I: As the first problem, finite difference method has been applied to 2D-CBE having the following exact solution over the region $\Omega=[0,1] \times[0,1][6]$

$$
\begin{aligned}
& u(x, y, t)=\frac{3}{4}-\frac{1}{4[1+\exp ((-4 x+4 y-t) \operatorname{Re} / 32]} \\
& v(x, y, t)=\frac{3}{4}+\frac{1}{4[1+\exp ((-4 x+4 y-t) \operatorname{Re} / 32]} .
\end{aligned}
$$

The initial and boundary conditions required for the application of the method are obtained from the analytical solution given by the equations (3.1)-(3.2). Table (1) presents the numerical solutions of Problem I for $u$ for values of $h_{x}=h_{y}=0.05, \operatorname{Re}=10, \Delta t=10^{-4}$ at times $t=0.01,0.5$ and 1.0. From the table it is clearly seen that both the 
Table 1. Numerical solutions of Problem I for $u$ for values of $h_{x}=h_{y}=0.05, \operatorname{Re}=10, \Delta t=10^{-4}$ at times $t=0.01$, 0.5 and 1.0 .

\begin{tabular}{clccccc}
\hline$(x, y)$ & \multicolumn{2}{c}{$t=0.01$} & \multicolumn{2}{c}{$t=0.5$} & \multicolumn{2}{c}{$t=1.0$} \\
\hline & \multicolumn{1}{c}{ Approx. } & Exact & Approx. & Exact & Approx. & Exact \\
\cline { 2 - 7 }$(0.1,0.1)$ & 0.624805 & 0.624805 & 0.615254 & 0.615254 & 0.605626 & 0.605626 \\
$(0.5,0.1)$ & 0.594202 & 0.594202 & 0.585396 & 0.585396 & 0.576840 & 0.576840 \\
$(0.9,0.1)$ & 0.567082 & 0.567082 & 0.559837 & 0.559837 & 0.553017 & 0.553017 \\
$(0.3,0.3)$ & 0.624805 & 0.624805 & 0.615255 & 0.615254 & 0.605627 & 0.605626 \\
$(0.7,0.3)$ & 0.594202 & 0.594202 & 0.585396 & 0.585396 & 0.576840 & 0.576840 \\
$(0.1,0.5)$ & 0.655431 & 0.655431 & 0.646276 & 0.646275 & 0.636685 & 0.636685 \\
$(0.5,0.5)$ & 0.624805 & 0.624805 & 0.615256 & 0.615254 & 0.605628 & 0.605626 \\
$(0.9,0.5)$ & 0.594202 & 0.594202 & 0.585396 & 0.585396 & 0.576840 & 0.576840 \\
$(0.3,0.7)$ & 0.655431 & 0.655431 & 0.646277 & 0.646275 & 0.636687 & 0.636685 \\
$(0.7,0.7)$ & 0.624805 & 0.624805 & 0.615256 & 0.615254 & 0.605629 & 0.605626 \\
$(0.1,0.9)$ & 0.682611 & 0.682611 & 0.674814 & 0.674814 & 0.666353 & 0.666353 \\
$(0.5,0.9)$ & 0.655431 & 0.655431 & 0.646277 & 0.646275 & 0.636687 & 0.636685 \\
$(0.9,0.9)$ & 0.624805 & 0.624805 & 0.615255 & 0.615254 & 0.605627 & 0.605626 \\
\hline$L_{2}$ & $8.419211 \times 10^{-8}$ & \multicolumn{3}{c}{$2.169158 \times 10^{-6}$} & & $2.354379 \times 10^{-6}$ \\
\hline$L_{\infty}$ & $6.693449 \times 10^{-8}$ & \multicolumn{3}{c}{$2.451640 \times 10^{-6}$} & & \\
\hline
\end{tabular}

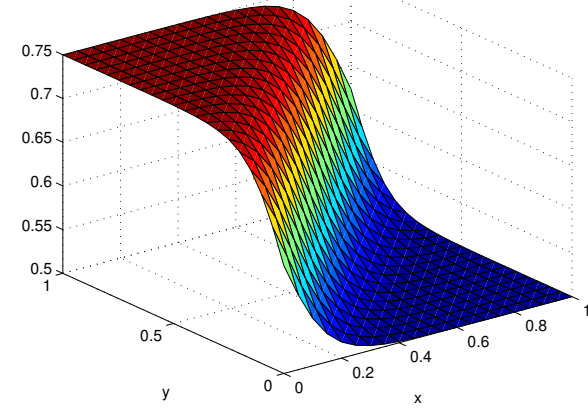

(a)

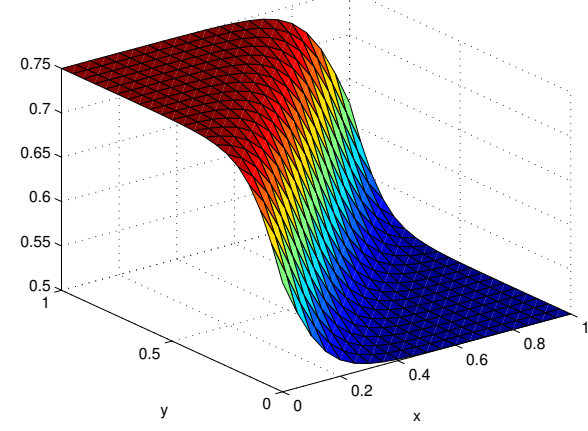

(b)

Figure 1. (a) Exact and (b) numerical solutions for $u$ of Problem 1 for values of $h_{x}=h_{y}=0.05, \operatorname{Re}=100, \Delta t=10^{-4}$ at $t=0.5$.

numerical and analytical solutions at selected points for each time level are very close to each other. Moreover, it is also seen that the computed error norms $L_{2}$ and $L_{\infty}$ are small enough to be acceptable. Table (2) presents the numerical solutions of Problem I for $v$ for values of $h_{x}=h_{y}=0.05$, Re $=10, \Delta t=10^{-4}$ at times $t=0.01,0.5$ and 1.0. Again from the table it can be observed that the numerical results are very close to their exact counterparts and computed error norms are small enough. Tables (3-4) show also pointwise values and the error norms $L_{2}$ and $L_{\infty}$ of $u$ and $v$ but now for a larger value of Reynold number Re $=100$, respectively. As it is seen from the tables, both of the error norms increase as the Reynold number increases. Figures (1-2) show first exact and then numerical solutions for $u$ and $v$ of Problem 1 for values of $h_{x}=h_{y}=0.05, \operatorname{Re}=100, \Delta t=10^{-4}$ at $t=0.0$, respectively.

Problem II: Rubin-Graves type linearization finite difference method has been applied to 2D-CBE on the solution domain $\Omega=[0,0.5] \times[0,0.5]$ with the following initial

$$
u(x, y, 0)=\sin \pi x+\cos \pi y, v(x, y, 0)=x+y
$$

and boundary conditions

$$
\begin{aligned}
& \left.\begin{array}{cc}
u(0, y, t)=\cos (\pi y), & u(0.5, y, t)=1+\cos (\pi y) \\
v(0, y, t)=y, & v(0.5, y, t)=0.5+y
\end{array}\right\} 0 \leq y \leq 0.5, t \geq 0 \\
& \left.\begin{array}{cc}
u(x, 0, t)=1+\sin (\pi x) & u(x, 0.5, t)=\sin (\pi x) \\
v(x, 0, t)=x & v(x, 0.5, t)=x+0.5
\end{array}\right\} 0 \leq x \leq 0.5, t \geq 0
\end{aligned}
$$


Table 2. Numerical solutions of Problem I for $v$ for values of $h_{x}=h_{y}=0.05, \operatorname{Re}=10, \Delta t=10^{-4}$ at times $t=0.01$, 0.5 and 1.0 .

\begin{tabular}{clccccc}
\hline$(x, y)$ & \multicolumn{2}{c}{$t=0.01$} & \multicolumn{2}{c}{$t=0.5$} & \multicolumn{2}{c}{$t=1.0$} \\
\hline & \multicolumn{1}{c}{ Approx. } & Exact & \multicolumn{1}{c}{ Approx. } & Exact & Approx. & Exact \\
\cline { 2 - 7 }$(0.1,0.1)$ & 0.875195 & 0.875195 & 0.884746 & 0.884746 & 0.894374 & 0.894374 \\
$(0.5,0.1)$ & 0.905798 & 0.905798 & 0.914604 & 0.914604 & 0.923160 & 0.923160 \\
$(0.9,0.1)$ & 0.932918 & 0.932918 & 0.940163 & 0.940163 & 0.946983 & 0.946983 \\
$(0.3,0.3)$ & 0.875195 & 0.875195 & 0.884745 & 0.884746 & 0.894373 & 0.894374 \\
$(0.7,0.3)$ & 0.905798 & 0.905798 & 0.914604 & 0.914604 & 0.923160 & 0.923160 \\
$(0.1,0.5)$ & 0.844569 & 0.844569 & 0.853724 & 0.853725 & 0.863315 & 0.863315 \\
$(0.5,0.5)$ & 0.875195 & 0.875195 & 0.884744 & 0.884746 & 0.894372 & 0.894374 \\
$(0.9,0.5)$ & 0.905798 & 0.905798 & 0.914604 & 0.914604 & 0.923160 & 0.923160 \\
$(0.3,0.7)$ & 0.844569 & 0.844569 & 0.853723 & 0.853725 & 0.863313 & 0.863315 \\
$(0.7,0.7)$ & 0.875195 & 0.875195 & 0.884744 & 0.884746 & 0.894371 & 0.894374 \\
$(0.1,0.9)$ & 0.817389 & 0.817389 & 0.825186 & 0.825186 & 0.833647 & 0.833647 \\
$(0.5,0.9)$ & 0.844569 & 0.844569 & 0.853723 & 0.853725 & 0.863313 & 0.863315 \\
$(0.9,0.9)$ & 0.875195 & 0.875195 & 0.884145 & 0.884146 & 0.894373 & 0.894374 \\
\hline$L_{2}$ & $6.013832 \times 10^{-8}$ & \multicolumn{3}{c}{$1.511454 \times 10^{-6}$} & & $2.804862 \times 10^{-6}$ \\
\hline$L_{\infty}$ & $6.693447 \times 10^{-8}$ & \multicolumn{3}{c}{$2.451640 \times 10^{-6}$} &
\end{tabular}

Table 3. Numerical solutions of Problem I for $u$ for values of $h_{x}=h_{y}=0.05, \operatorname{Re}=100, \Delta t=10^{-4}$ at times $t=0.01$, 0.5 and 1.0 .

\begin{tabular}{|c|c|c|c|c|c|c|}
\hline$(x, y)$ & \multicolumn{2}{|c|}{$t=0.01$} & \multicolumn{2}{|c|}{$t=0.5$} & \multicolumn{2}{|c|}{$t=2.0$} \\
\hline & Approx. & Exact & Approx. & Exact & Approx. & Exact \\
\hline$(0.1,0.1)$ & 0.623106 & 0.623047 & 0.543002 & 0.543322 & 0.500470 & 0.500482 \\
\hline$(0.5,0.1)$ & 0.501617 & 0.501622 & 0.500341 & 0.500353 & 0.500003 & 0.500003 \\
\hline$(0.9,0.1)$ & 0.500011 & 0.500011 & 0.500002 & 0.500002 & 0.500000 & 0.500000 \\
\hline$(0.3,0.3)$ & 0.623106 & 0.623047 & 0.642692 & 0.543322 & 0.500441 & 0.500482 \\
\hline$(0.7,0.3)$ & 0.501617 & 0.501622 & 0.500317 & 0.500353 & 0.500003 & 0.500003 \\
\hline$(0.1,0.5)$ & 0.748272 & 0.748274 & 0.742150 & 0.742214 & 0.555153 & 0.555675 \\
\hline$(0.5,0.5)$ & 0.623106 & 0.623047 & 0.542509 & 0.543322 & 0.500414 & 0.500482 \\
\hline$(0.9,0.5)$ & 0.501617 & 0.501622 & 0.500304 & 0.500353 & 0.500003 & 0.500003 \\
\hline$(0.3,0.7)$ & 0.748272 & 0.748274 & 0.742114 & 0.742214 & 0.554816 & 0.555675 \\
\hline$(0.7,0.7)$ & 0.623106 & 0.623047 & 0.542463 & 0.543322 & 0.500384 & 0.500482 \\
\hline$(0.1,0.9)$ & 0.749988 & 0.749988 & 0.749945 & 0.749946 & 0.744196 & 0.744256 \\
\hline$(0.5,0.9)$ & 0.748272 & 0.748274 & 0.742103 & 0.742214 & 0.554504 & 0.555675 \\
\hline$(0.9,0.9)$ & 0.623106 & 0.623047 & 0.542282 & 0.543322 & 0.500525 & 0.500482 \\
\hline$L_{2}$ & $3.811712 \times 10^{-5}$ & & $1.070747 \times 10^{-3}$ & & $1.097702 \times 10^{-3}$ & \\
\hline$L_{\infty}$ & $6.071263 \times 10^{-5}$ & & $2.031654 \times 10^{-3}$ & & $2.240898 \times 10^{-3}$ & \\
\hline
\end{tabular}

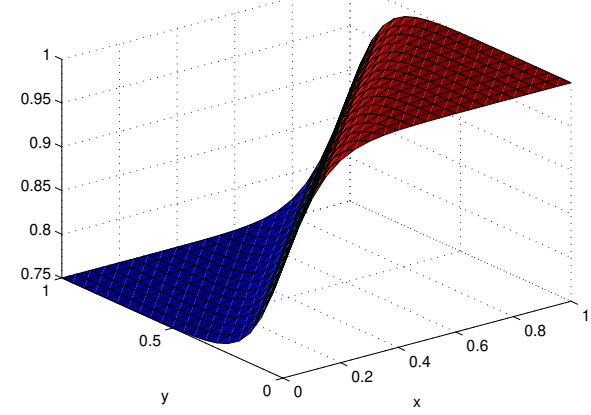

(a)

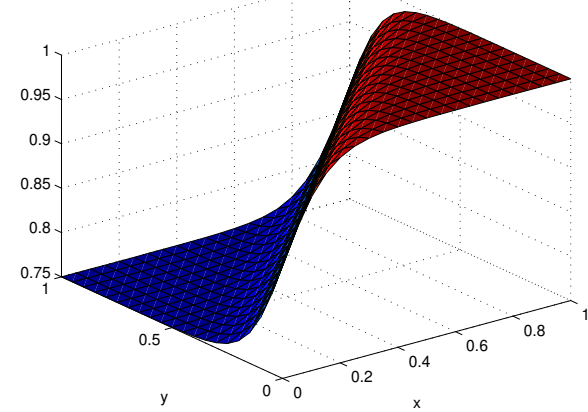

(b)

Figure 2. (a) Exact and (b) numerical solutions for $v$ of Problem 1 for values of $h_{x}=h_{y}=0.05, \operatorname{Re}=100, \Delta t=10^{-4}$ at $t=0.5$. 
Table 4. Numerical solutions of Problem I for $v$ for values of $h_{x}=h_{y}=0.05, \operatorname{Re}=100, \Delta t=10^{-4}$ at times $t=0.01$, 0.5 and 1.0 .

\begin{tabular}{clclccc}
\hline$(x, y)$ & \multicolumn{2}{c}{$t=0.01$} & \multicolumn{2}{c}{$t=0.5$} & \multicolumn{2}{c}{$t=2.0$} \\
\hline & \multicolumn{1}{c}{ Approx. } & Exact & \multicolumn{1}{c}{ Approx. } & Exact & Approx. & Exact \\
\cline { 2 - 7 }$(0.1,0.1)$ & 0.876894 & 0.876953 & 0.956998 & 0.956678 & 0.999530 & 0.999518 \\
$(0.5,0.1)$ & 0.998383 & 0.998378 & 0.999659 & 0.999647 & 0.999997 & 0.999997 \\
$(0.9,0.1)$ & 0.999989 & 0.999989 & 0.999998 & 0.999998 & 1.000000 & 1.000000 \\
$(0.3,0.3)$ & 0.876894 & 0.876953 & 0.957308 & 0.956678 & 0.999559 & 0.999518 \\
$(0.7,0.3)$ & 0.998383 & 0.998378 & 0.999683 & 0.999647 & 0.999997 & 0.999997 \\
$(0.1,0.5)$ & 0.751728 & 0.751726 & 0.757850 & 0.757786 & 0.944847 & 0.944325 \\
$(0.5,0.5)$ & 0.876894 & 0.876953 & 0.957491 & 0.956678 & 0.999586 & 0.999518 \\
$(0.9,0.5)$ & 0.998383 & 0.998378 & 0.999696 & 0.999647 & 0.999997 & 0.999997 \\
$(0.3,0.7)$ & 0.751728 & 0.751726 & 0.757886 & 0.757786 & 0.945184 & 0.944325 \\
$(0.7,0.7)$ & 0.876894 & 0.876953 & 0957537 & 0.956678 & 0.999616 & 0.999518 \\
$(0.1,0.9)$ & 0.750012 & 0.750012 & 0.750055 & 0.750054 & 0.755804 & 0.755744 \\
$(0.5,0.9)$ & 0.751728 & 0.751726 & 0.757897 & 0.757786 & 0.945496 & 0.944325 \\
$(0.9,0.9)$ & 0.876894 & 0.876953 & 0.957718 & 0.956678 & 0.999475 & 0.999518 \\
\hline$L_{2}$ & $2.736786 \times 10^{-5}$ & \multicolumn{5}{c}{$7.126002 \times 10^{-4}$} \\
\hline$L_{\infty}$ & $6.071263 \times 10^{-5}$ & \multicolumn{3}{c}{$6.043011 \times 10^{-4}$} & \\
\hline
\end{tabular}

Table 5. A comparison of numerical solutions for $u$ of Problem 2 for values of $h_{x}=h_{y}=0.025, \operatorname{Re}=500, \Delta t=10^{-4}$ at time $t=0.625, N=40$ with those in Refs. [5, 6, 12].

\begin{tabular}{cccccc}
\hline$(x, y)$ & \multicolumn{5}{c}{$u$} \\
\hline & Present & {$[5]$} & {$[5] \mathrm{N}=40$} & {$[6]$} & {$[12]$} \\
\cline { 2 - 6 }$(0.15,0.1)$ & 0.96870 & 0.95691 & 0.96066 & 0.96650 & 0.96870 \\
$(0.3,0.1)$ & 1.03204 & 0.95616 & 0.96852 & 1.02970 & 1.03200 \\
$(0.1,0.2)$ & 0.84618 & 0.84257 & 0.84104 & 0.84449 & 0.86178 \\
$(0.2,0.2)$ & 0.87813 & 0.86399 & 0.86866 & 0.87631 & 0.87813 \\
$(0.1,0.3)$ & 0.67920 & 0.67667 & 0.67792 & 0.67809 & 0.67920 \\
$(0.3,0.3)$ & 0.79944 & 0.76876 & 0.77254 & 0.79792 & 0.79945 \\
$(0.15,0.4)$ & 0.54675 & 0.54408 & 0.54543 & 0.54601 & 0.66039 \\
$(0.2,0.4)$ & 0.58958 & 0.58778 & 0.58564 & 0.58874 & 0.58958 \\
\hline
\end{tabular}

[12]. There is no analytical solution to this problem. Since Problem II has not analytical solution in Table (5), a comparison of numerical solutions for $u$ of Problem 2 for values of $h_{x}=h_{y}=0.025, \operatorname{Re}=500, \Delta t=10^{-4}$ at time $t=0.625, N=40$ with those in Refs. [5, 6, 12] is presented. Again, due to the same reason, Table (6) presents a comparison of numerical solutions for $v$ of Problem 2 for values of $h_{x}=h_{y}=0.025, \operatorname{Re}=500, \Delta t=10^{-4}$ at time $t=0.625$ with those in Refs. [5, 6, 12]. Tables (7-8) show also pointwise values of $u$ and $v$ but now for a smaller value of Reynold number $\operatorname{Re}=50$, respectively. Figures (3) shows numerical solutions of $u$ and $v$ of Problem 2 for values of $h_{x}=h_{y}=0.025, \operatorname{Re}=50, \Delta t=10^{-4}$ at time $t=0.625$, respectively.

Problem III: The solution domain of the third problem is $\Omega=\{(x, y): 0 \leq x \leq 1,0 \leq y \leq 1\}$ and its analytical solution is of the form [12]

$$
\begin{aligned}
& u(x, y, t)=-\frac{4 \pi e^{-\frac{5 \pi^{2} t}{\mathrm{Re}}} \cos (2 \pi x) \sin (\pi y)}{\operatorname{Re}\left(2+e^{-\frac{5 \pi^{2} t}{\mathrm{Re}}} \sin (2 \pi x) \sin (\pi y)\right.} \\
& v(x, y, t)=-\frac{2 \pi e^{-\frac{5 \pi^{2} t}{\mathrm{Re}}} \sin (2 \pi x) \cos (\pi y)}{\operatorname{Re}\left(2+e^{-\frac{5 \pi^{2} t}{\mathrm{Re}}} \sin (2 \pi x) \sin (\pi y)\right.}
\end{aligned}
$$

Table (9) presents numerical solutions of $u$ of Problem 3 for values of $h_{x}=h_{y}=0.05$, $\operatorname{Re}=1000, \Delta t=10^{-3}$ at times $t=0.01,0.5$ and 1.0. From the table one can easily see that the approximate and exact solutions are very close to each other and calculated error norms $L_{2}$ and $L_{\infty}$ are small enough. In a similar manner, Table (10) presents numerical solutions of $v$ of Problem 3 for values of $h_{x}=h_{y}=0.05, \operatorname{Re}=1000, \Delta t=10^{-3}$ at times $t=0.01,0.5$ and 1.0. Again, one can see from this table that both of the approximate and exact pointwise values are in good agreement. Th error norms $L_{2}$ and $L_{\infty}$ show the general consistency between the approximate and exact solutions 
Table 6. A comparison of numerical solutions for $v$ of Problem 2 for values of $h_{x}=h_{y}=0.025, \operatorname{Re}=500, \Delta t=10^{-4}$ at time $t=0.625, N=40$ with those in Refs. [5, 6, 12].

\begin{tabular}{ccclcc}
\hline$(x, y)$ & \multicolumn{5}{c}{$v$} \\
\hline & Present & {$[5]$} & {$[5] \mathrm{N}=40$} & {$[6]$} & {$[12]$} \\
\cline { 2 - 6 }$(0.15,0.1)$ & 0.09044 & 0.10177 & 0.08612 & 0.09020 & 0.09043 \\
$(0.3,0.1)$ & 0.10730 & 0.13287 & 0.07712 & 0.10690 & 0.10728 \\
$(0.1,0.2)$ & 0.18010 & 0.18503 & 0.17828 & 0.17972 & 0.17295 \\
$(0.2,0.2)$ & 0.16816 & 0.18169 & 0.16202 & 0.16777 & 0.16816 \\
$(0.1,0.3)$ & 0.26268 & 0.26560 & 0.26094 & 0.26222 & 0.26268 \\
$(0.3,0.3)$ & 0.23550 & 0.25142 & 0.21542 & 0.23497 & 0.23550 \\
$(0.15,0.4)$ & 0.31799 & 0.32084 & 0.31360 & 0.31753 & 0.29022 \\
$(0.2,0.4)$ & 0.30418 & 0.30927 & 0.29776 & 0.30371 & 0.30418 \\
\hline
\end{tabular}

Table 7. A comparison of numerical solutions for $u$ of Problem 2 for values of $h_{x}=h_{y}=0.025, \operatorname{Re}=50, \Delta t=10^{-4}$ at time $t=0.625$ with those in Refs. [5, 6, 12].

\begin{tabular}{cllll}
\hline$(x, y)$ & \multicolumn{4}{c}{$u$} \\
\hline & Present & {$[5]$} & {$[6]$} & {$[12]$} \\
\cline { 2 - 5 }$(0.1,0.1)$ & 0.97146 & 0.97258 & 0.96688 & 0.97146 \\
$(0.3,0.1)$ & 1.15282 & 1.16214 & 1.14827 & 1.15280 \\
$(0.2,0.2)$ & 0.86308 & 0.86281 & 0.85911 & 0.86308 \\
$(0.4,0.2)$ & 0.97984 & 0.96483 & 0.97637 & 0.97984 \\
$(0.1,0.3)$ & 0.66316 & 0.66318 & 0.66019 & 0.66316 \\
$(0.3,0.3)$ & 0.77232 & 0.77030 & 0.76932 & 0.77232 \\
$(0.2,0.4)$ & 0.58181 & 0.58070 & 0.57966 & 0.58181 \\
$(0.4,0.4)$ & 0.75861 & 0.74435 & 0.75678 & 0.75860 \\
\hline
\end{tabular}

Table 8. A comparison of numerical solutions for $v$ of Problem 2 for values of $h_{x}=h_{y}=0.025, \operatorname{Re}=50, \Delta t=10^{-4}$ at time $t=0.625$ with those in Refs. [5, 6, 12].

\begin{tabular}{cllll}
\hline$(x, y)$ & \multicolumn{4}{c}{$v$} \\
\hline & Present & {$[5]$} & {$[6]$} & {$[12]$} \\
\cline { 2 - 5 }$(0.1,0.1)$ & 0.09869 & 0.09773 & 0.09824 & 0.09869 \\
$(0.3,0.1)$ & 0.14158 & 0.14039 & 0.14112 & 0.14158 \\
$(0.2,0.2)$ & 0.16754 & 0.16660 & 0.16681 & 0.16754 \\
$(0.4,0.2)$ & 0.17110 & 0.17397 & 0.17065 & 0.17110 \\
$(0.1,0.3)$ & 0.26378 & 0.26294 & 0.26261 & 0.26378 \\
$(0.3,0.3)$ & 0.22654 & 0.22463 & 0.22576 & 0.22655 \\
$(0.2,0.4)$ & 0.32851 & 0.32402 & 0.32745 & 0.32851 \\
$(0.4,0.4)$ & 0.32500 & 0.31822 & 0.32441 & 0.32501 \\
\hline
\end{tabular}

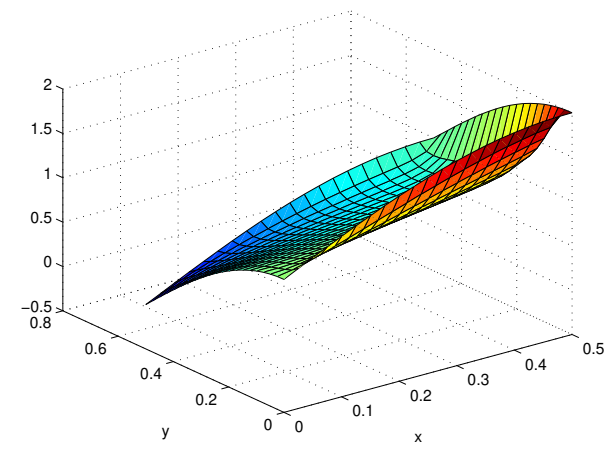

(a)

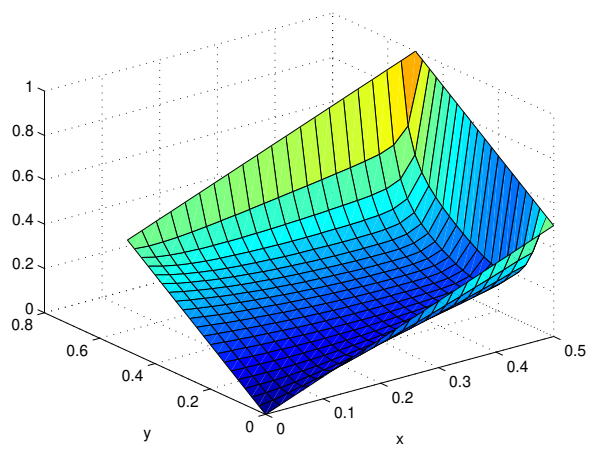

(b)

Figure 3. Numerical solutions of (a) $u$ and (b) $v$ of Problem 2 for values of $h_{x}=h_{y}=0.025, \operatorname{Re}=50, \Delta t=10^{-4}$ at time $t=0.625$. 
Table 9. Numerical solutions of $u$ of Problem 3 for values of $h_{x}=h_{y}=0.05, \operatorname{Re}=1000, \Delta t=10^{-3}$ at times $t=0.01$, 0.5 and 1.0 .

\begin{tabular}{|c|c|c|c|c|c|c|}
\hline$(x, y)$ & \multicolumn{2}{|c|}{$t=0.01$} & \multicolumn{2}{|c|}{$t=0.5$} & \multicolumn{2}{|c|}{$t=1.0$} \\
\hline & Approx. & Exact & Approx. & Exact & Approx. & Exact \\
\hline$(0.1,0.1)$ & -0.001439 & -0.001439 & -0.001408 & -0.001408 & -0.001376 & -0.001376 \\
\hline$(0.5,0.1)$ & 0.001941 & 0.001941 & 0.001895 & 0.001894 & 0.001849 & 0.001848 \\
\hline$(0.9,0.1)$ & -0.001727 & -0.001727 & -0.001682 & -0.001682 & -0.001638 & -0.001637 \\
\hline$(0.3,0.3)$ & 0.001134 & 0.001134 & 0.001114 & 0.001114 & 0.001094 & 0.001094 \\
\hline$(0.7,0.3)$ & 0.002551 & 0.002551 & 0.002458 & 0.002453 & 0.002368 & 0.002359 \\
\hline$(0.1,0.5)$ & -0.003927 & -0.003927 & -0.003854 & -0.003854 & -0.003780 & -0.003781 \\
\hline$(0.5,0.5)$ & 0.006280 & 0.006280 & 0.006130 & 0.006130 & 0.005981 & 0.005981 \\
\hline$(0.9,0.5)$ & -0.007194 & -0.007194 & -0.006960 & -0.006953 & -0.006731 & -0.006718 \\
\hline$(0.3,0.7)$ & 0.001134 & 0.001134 & 0.001114 & 0.001114 & 0.001094 & 0.001094 \\
\hline$(0.7,0.7)$ & 0.002551 & 0.002551 & 0.002458 & 0.002453 & 0.002368 & 0.002359 \\
\hline$(0.1,0.9)$ & -0.001439 & -0.001439 & -0.001408 & -0.001408 & -0.001376 & -0.001376 \\
\hline$(0.5,0.9)$ & 0.001941 & 0.001941 & 0.001895 & 0.001894 & 0.001849 & 0.001848 \\
\hline$(0.9,0.9)$ & -0.001727 & -0.001727 & -0.001682 & -0.001682 & -0.001638 & -0.001637 \\
\hline$L_{2}$ & $2.2105 \times 10^{-5}$ & & $1.0312 \times 10^{-3}$ & & $1.9287 \times 10^{-3}$ & \\
\hline$L_{\infty}$ & $2.8241 \times 10^{-7}$ & & $1.2663 \times 10^{-5}$ & & $2.2938 \times 10^{-5}$ & \\
\hline
\end{tabular}

throughout the solution domain. Figures (4-5) show first exact and then numerical solutions for $u$ and $v$ of Example 3 for values of $h_{x}=h_{y}=0.05, \operatorname{Re}=1000, \Delta t=10^{-3}$ at $t=0.01$, respectively.

Table 10. Numerical solutions of $v$ of Problem 3 for values of $h_{x}=h_{y}=0.05, \operatorname{Re}=1000, \Delta t=10^{-3}$ at times $t=0.01,0.5$ and 1.0 .

\begin{tabular}{crrrrrr}
\hline$(x, y)$ & \multicolumn{2}{c}{$t=0.01$} & \multicolumn{2}{c}{$t=1.0$} \\
\hline & Approx. & \multicolumn{1}{c}{ Exact } & Approx. & \multicolumn{1}{c}{ Exact } & Approx. & \multicolumn{1}{c}{ Ecaxt } \\
\cline { 2 - 7 }$(0.1,0.1)$ & -0.001609 & -0.001609 & -0.001574 & -0.001574 & -0.001539 & -0.001539 \\
$(0.5,0.1)$ & -0.000000 & -0.000000 & -0.000000 & -0.000000 & -0.000001 & -0.000000 \\
$(0.9,0.1)$ & 0.001931 & 0.001931 & 0.001880 & 0.001880 & 0.001830 & 0.001830 \\
$(0.3,0.3)$ & -0.001268 & -0.001268 & -0.001246 & -0.001246 & -0.001223 & -0.001224 \\
$(0.7,0.3)$ & 0.002852 & 0.002852 & 0.002746 & 0.002743 & 0.002643 & 0.002637 \\
$(0.1,0.5)$ & -0.000000 & -0.000000 & -0.000000 & -0.000000 & -0.000000 & -0.000000 \\
$(0.5,0.5)$ & -0.000000 & -0.000000 & 0.000000 & -0.000000 & -0.000000 & -0.000000 \\
$(0.9,0.5)$ & 0.000000 & 0.000000 & 0.000000 & 0.000000 & 0.000000 & 0.000000 \\
$(0.3,0.7)$ & 0.001268 & 0.001268 & 0.001246 & 0.001246 & 0.001223 & 0.001224 \\
$(0.7,0.7)$ & -0.002852 & -0.002852 & -0.002746 & -0.002743 & -0.002643 & -0.002637 \\
$(0.1,0.9)$ & 0.001609 & 0.001609 & 0.001574 & 0.001574 & 0.001539 & 0.001539 \\
$(0.5,0.9)$ & 0.000000 & 0.000000 & 0.000000 & 0.000000 & 0.000001 & 0.000000 \\
$(0.9,0.9)$ & -0.001931 & -0.001931 & -0.001880 & -0.001880 & -0.001830 & -0.001830 \\
\hline$L_{2}$ & $1.2846 \times 10^{-5}$ & \multicolumn{5}{c}{$6.0214 \times 10^{-4}$} \\
\hline$L_{\infty}$ & $9.3390 \times 10^{-8}$ & $4.1431 \times 10^{-6}$ & & $7.1320 \times 10^{-3}$ & \\
\hline
\end{tabular}




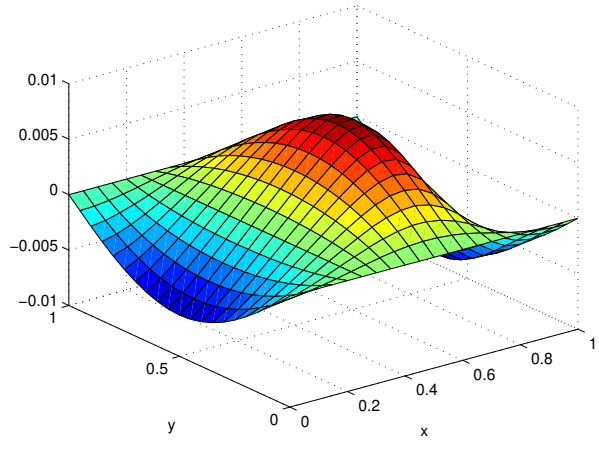

(a)

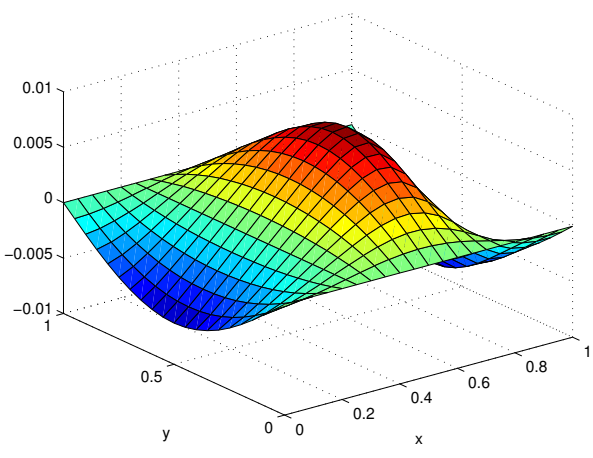

(b)

Figure 4. (a) Exact and (b) numerical solutions of $u$ of Problem 3 for values $h_{x}=h_{y}=0.05, \operatorname{Re}=1000, \Delta t=10^{-3}$ at time $t=0.01$.

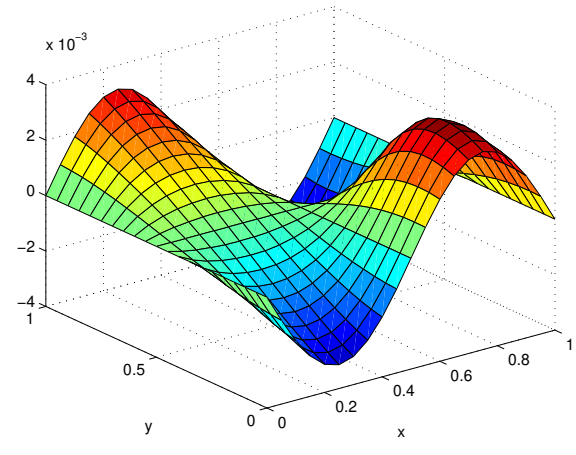

(a)

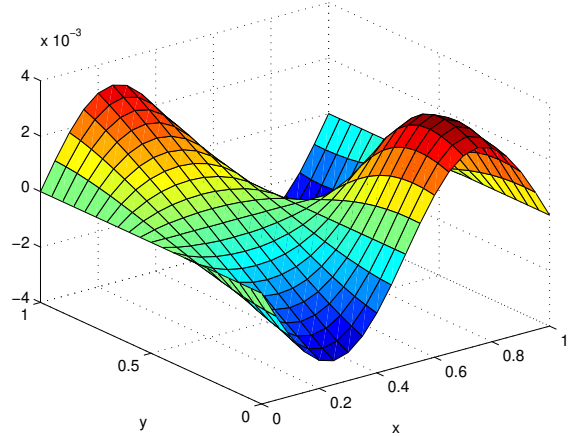

(b)

Figure 5. (a) Exact and (b) numerical solutions of $v$ of Problem 3 for values $h_{x}=h_{y}=0.05, \operatorname{Re}=1000, \Delta t=10^{-3}$ at time $t=0.01$.

\section{Conclusion}

In this study, numerical solutions of two dimensional coupled Burgers equation has been obtained by using finite difference method based on a Rubin-Graves type linearization. To demonstrate the accuracy and efficiency of the method, this method has been applied to three test problems with known analytical solutions and to one test problem with unknown analytical solution. The error norms $L_{2}$ and $L_{\infty}$ have been calculated. From these calculations, it is seen that the proposed method yield good enough results, and it is simple and easy to apply. In conclusion, numerical solution of two dimensional coupled nonlinear partial differential equations arises in physical sciences can be achieved easily and effectively by the proposed method. The algebraic systems found out by using the proposed schemes can be easily stored and solved by the software systems of nowadays. As a conclusion, the proposed method can be easily and successfully applied to this type of problems arising in applied mathematics, mathematical physics and engineering science.

\section{Funding}

There is no funding for this work.

\section{Availability of data and materials}

Not applicable. 


\section{Competing interests}

The authors declare that they have no competing interests.

\section{Author's contributions}

All authors contributed equally to the writing of this paper. All authors read and approved the final manuscript.

\section{References}

[1] Fletcher, C. A. J., Generating exact solutions of the two-dimensional Burgers' equations, Int. J. for Numer. Meth. Fluids, 3 (1983),3, pp. 213-216. https:/ /doi.org/10.1002/fld.1650030302

[2] C. A.J. Fletcher, A comparison of finite element and finite difference solutions of the one- and two-dimensional Burgers' equations, Journal of Computational Physics, 51(1), 159-188 (1983). https:/ / doi.org/10.1016/00219991(83)90085-2

[3] Goyon, O., Multilevel Schemes for Solving Unsteady Equations, Int. J. Numer. Meth. Fluids, 22 (1996), 10, pp. 937-959

[4] Arshed Ali , Siraj-ul-Islam and Sirajul Haq (2009) A Computational Meshfree Technique for the Numerical Solution of the Two-Dimensional Coupled Burgers' Equations, International Journal for Computational Methods in Engineering Science and Mechanics, 10:5, 406-422. https:/ / doi.org/10.1080/15502280903108016

[5] Jain, P. C., Holla, D. N., Numerical solutions of coupled Burgers' equation, Int. J. Numer. Meth., 13 (1978), 4, pp. 213-222. https://doi.org/10.1016/0020-7462(78)90024-0

[6] Bahadır, A. R. A fully implicit finite-difference scheme for two-dimensional Burgers' equations, Applied Mathematics and Computation, Applied Mathematics and Computation 137 (2003) 131-137. https://doi.org/10.1016/S0096-3003(02)00091-7

[7] A.H. Khater, R.S. Temsah, M.M. Hassan, A Chebyshev spectral collocation method for solving Burgers'-type equations, Journal of Computational and Applied Mathematics 222 (2008) 333-350. https://doi.org/10.1016/j.cam.2007.11.007

[8] R. C. Mittal and Ram Jiwari, Differential Quadrature Method for Two-Dimensional Burgers' Equations, International Journal for Computational Methods in Engineering Science and Mechanics, 10:450-459, 2009. DOI: $10.1080 / 15502280903111424$

[9] W. Liao, A fourth-order finite-difference method for solving the system of two-dimensional Burgers' equations, Int. J. Numer. Meth. Fluids 2010; 64:565-590. https://doi.org/10.1002/fld.2163

[10] H. Zhu, H. Shu, M. Ding, Numerical solutions of two-dimensional Burgers' equations by discrete Adomian decomposition method, Computers and Mathematics with Applications 60 (2010) 840-848. https://doi.org/10.1016/j.camwa.2010.05.031

[11] V. K. Srivastava, M. Tamsir, U. Bhardwaj, YVSS Sanyasiraju, Crank-Nicolson Scheme for Numerical Solutions of Two-dimensional Coupled Burgers' Equations, International Journal of Scientific \& Engineering Research 2(5), pp1-6 May-2011

[12] Mohammad Tamsir, Vineet Kumar Srivastava, A semi-implicit finite-difference approach for two-dimensional coupled Burgers equations, International Journal of Scientific \& Engineering Research, 2(6), pp. 46-51, June-2011, ISSN 2229-5518

[13] V. K. Srivastava, M. Tamsir, Crank-Nicolson Semi-Implicit Approach For Numerical Solutions of Two- Dimensional Coupled Nonlinear Burgers Equations, Int. J. of Applied Mechanics and Engineering, 2012, 17(2), pp.571-581

[14] S. Thakar and S.Wani, Linear Method For Two Dimensional Burgers Equation,Ultra Scientist Vol. 25(1)A, 156-168 (2013). 
[15] Vineet K. Srivastava, Mukesh K. Awasthi, and Sarita Singh, An implicit logarithmic finite-difference technique for two dimensional coupled viscous Burgers' equation, AIP Advances 3, 122105 (2013); doi: 10.1063/1.4842595

[16] Vineet K. Srivastava, Sarita Singh, and Mukesh K. Awasthi, Numerical solutions of coupled Burgers equations by an implicit finite difference scheme, AIP Advances 3(8), 082131 (2013); doi: 10.1063/1.4820355

[17] Vineet K. Srivastava and Brajesh Kumar Singh, A robust finite difference scheme for the numerical solutions of two dimensional time dependent coupled nonlinear Burgers equations, Int. J. of Appl. Math and Mech. 10 (7): 28-39, 2014.

[18] L. Zhang, L. Wang and X. Ding, Exact finite-difference scheme and nonstandard finite-difference scheme for coupled Burgers equation, Advances in Difference Equations 2014, 2014:122. DOI: 10.1186/1687-1847-2014-122

[19] R C Mittal Amit Tripathi , (2015), Numerical solutions of two-dimensional Burgers' equations using modified Bi-cubic B-spline finite elements, Engineering Computations, Vol. 32 Iss 5 pp. 1275 - 1306. https://doi.org/10.1108/EC-04-2014-0067

[20] Mohammad Tamsir, VineetK. Srivastava, Ram Jiwari, An algorithm based on exponential modified cubic Bspline differential quadrature method for nonlinear Burgers' equation, Applied Mathematics and Computation, 290 (2016) 111-124. https:/ / doi.org/10.1016/j.amc.2016.05.048

[21] T. Zhanlav, O. Chuluunbaatar, V. Ulziibayar, Higher-Order Numerical Solution of Two-Dimensional Coupled Burgers Equations, American Journal of Computational Mathematics, 2016, 6, 120-129. DOI: 10.4236/ajcm.2016.62013

[22] Ngondiep E. An efficient three-level explicit time-split scheme for solving two-dimensional unsteady nonlinear coupled Burgers' equations. Int J Numer Meth Fluids. 2020;92:266-284. https://doi.org/10.1002/fld.4783

[23] M. Saqib, S. Hasnain and D. S. Mashat, Highly Efficient Computtaional Methods for Two Dimensional Coupled Nonlinear Unsteady Convection-Diffusion Problems, IEEE Access, Vol.5, 2017. DOI: 10.1109/ ACCESS.2017.2699320

[24] F.W. Wubs and E.D. de Goede, An explicit-implicit method for a class of time-dependent partial differential equations, Appl. Numer. Math., 9 (1992) 157-181. https:/ / doi.org/10.1016/0168-9274(92)90012-3

[25] Y. Chai, J. Ouyang, Appropriate stabilized Galerkin approaches for solving two-dimensional coupled Burgers' equations at high Reynolds numbers, Computers and Mathematics with Applications 79 (2020) 1287-1301. https://doi.org/10.1016/j.camwa.2019.08.036

[26] S.G. Rubin and R.A. Graves, A Cubic Spline Approximation for Problems in Fluid Mechanics, NASA, Washington, D.C., October, 1975.

[27] H.S. Shukla, M. Tamsir, V.K. Srivastava, J. Kumar, Numerical Solution of two dimensional coupled viscous Burgers' Equation using the Modified Cubic B-Spline Differential Quadrature Method, ArciheX. DOI: 10.1063/1.4902507.

\section{Affiliations}

NURI MURAT YAĞMURLU

AdDrESS: İnönü University, Dept. of Mathematics, 44200, Malatya-TURKEY.

E-MAIL: murat.yagmurlu@inonu.edu.tr

ORCID ID: 0000-0003-1593-0254

ABDULNASIR GAGIR

ADDRESS: İnönü University, Dept. of Mathematics, 44200, Malatya-TURKEY.

E-MAIL: ansrggr@gmail.com

ORCID ID: 0000-0003-1029-4447 